\title{
Early detection: a long road ahead
}

\author{
Recent data on blood-based tests for early cancer detection are promising, but many issues \\ must be addressed before we have clinically useful blood-based screening tests.
}

Evidence from at least some cancer types (including cervical and colon cancers ${ }^{1}$ ) suggests that earlier detection of cancer will improve survival. Despite the apparent need, then, to improve screening of the general population for incipient cancers, progress in this field has been fairly limited, and we still have only a handful of cancer screening tests that have clinical value. Recent data have given a glimmer of hope that blood-based ('liquid biopsy') cancer screening tests for many different cancer types are on the horizon, and the news stories on these data have brought early cancer detection back in the public eye. We agree these data are promising, but there are still many challenges ahead.

Cohen et al. ${ }^{2}$ took a minimalist approach, aiming to create a cancer screening test that was cost effective and could be conducted in a high-throughput manner on a large number of samples. The resulting CancerSEEK test analyses both genetic and protein biomarkers from blood samples using PCR-based sequencing of 61 amplicons from 16 cancer-relevant genes in cell-free DNA (cfDNA) and the expression levels of 8 proteins in plasma. Of 1005 patients who had been recently diagnosed with one of eight types of non-metastatic cancer, the test was able to identify the presence of a tumour (sensitivity) in 33-98\% of cases, and in those identified to have a tumour, its tissue of origin was correctly narrowed to two anatomical sites in $83 \%$ of cases. By contrast, only 7 of 812 healthy controls had a positive test.

At the recent American Association for Cancer Research Annual Meeting, Aravanis et al. ${ }^{3}$ reported initial data from the Circulating Cell-free Genome Atlas Study being conducted by GRAIL. The data are preliminary but indicate the potential of a broad-spectrum test conducted on cfDNA (using targeted sequencing of a 507 gene panel, whole-genome sequencing and methylation analysis by bisulfite sequencing). A key part of their analysis was removing the 'noise' from mutations arising from clonal haematopoiesis; to do this, they subtracted out mutations from white blood cell-derived cfDNA. In patients previously diagnosed with one of five highmortality cancers, sensitivities ranged from 50-95\%, depending on the assay or cancer stage ${ }^{4}$. Only 5 of 580 controls had a positive test, but since then, two of those have been diagnosed with a tumour.

Both tests were able to detect cancer in some patients who already had symptomatic disease, but there was wide variability in the sensitivities achieved. This is clearly a hurdle that must be overcome, especially as for a screening test to have clinical utility to detect presymptomatic tumours, it will need to be able to detect a much smaller signal. That two control patients in the GRAIL analysis who had a positive test were later diagnosed with cancer suggests that early detection could have been achieved in these patients. However, these are only anecdotal cases, and large-scale studies evaluating the performance of early detection tests in a real-world setting will be necessary.

Both tests report specificities $>99 \%$. Although high specificity is important to ensure low numbers of false positives, in an unscreened general population, where disease prevalence is low, this will still result in a substantial number of false positives ${ }^{5}$.

There are additional issues hinted at with these studies that will need to be addressed. First, we need to be able to determine the site of origin of a tumour to ensure the most appropriate follow-up after a positive signal. Driver mutations are typically not tissue-specific, so additional parameters (such as protein or methylation biomarkers) will need to be considered. We also need to avoid over-treatment of benign lesions that would never have progressed or harmed the patient; to achieve this, we will need more research into the biology and evolution of cancer progression in every tissue type.

Initially, it seems likely that blood-based screening tests will be most useful in populations already identified as being high risk (for example, smokers or those carrying germline mutations that increase cancer risk), where disease prevalence is higher. However, even in these cases, there is a long road ahead for such tests to prove their clinical value, and concerted research efforts will be important for meaningful and rapid progress.

1. Cho, $\mathrm{H}$. et al. When do changes in cancer survival mean progress? The insight from population incidence and mortality. J. Natl Cancer Inst. Monogr. 2014, 187-197 (2014).

2. Cohen, J. D. et al. Detection and localization of surgically resectable cancers with a multi-analyte blood test. Science 359, 926-930 (2018).

3. Aravanis, A. A. et al. in Proceedings of the 109th Annual Meeting of the American Association for Cancer Research. LB-343 (Chicago, IL, 2018).

4. [No authors listed.] GRAIL announces data from prototype blood tests for early cancer detection. Business Wire https://www. businesswire.com/news/home/20180417006580/en/GRAILAnnounces-Data-Prototype-Blood-Tests-Early (2018).

5. Kalinich, M. \& Haber, D. A. Cancer detection: seeking signals in blood. Science 359, 866-867 (2018). 